\title{
A 2-group construction from an extension of the 3-loop group $\Omega^{3} G$
}

\author{
Jouko Mickelsson ${ }^{1} \cdot$ Ossi Niemimäki ${ }^{1}$
}

Received: 11 October 2018 / Revised: 26 June 2019 / Accepted: 8 July 2019 / Published online: 22 July 2019

(c) The Author(s) 2019

\begin{abstract}
We define a 3-loop group $\Omega^{3} G$ as a subgroup of smooth maps from a 3-ball to a Lie group $G$, and then construct a 2 -group based on an automorphic action on the Mickelsson-Faddeev extension of $\Omega^{3} G$. In this, we follow the strategy of Murray et al. (J Lie Theory 27(4):1151-1177, 2017), who earlier described a similar construction for one-dimensional loops. The three-dimensional situation presented here is further complicated by the fact that the 3-loop group extension is not central.
\end{abstract}

Keywords Loop group · Lie 2-group · Mickelsson-Faddeev extension

Mathematics Subject Classification 22E67 $\cdot 81 \mathrm{R} 10 \cdot 18 \mathrm{D} 35$

\section{Introduction}

There is a growing interest in generalizing Lie groups and algebras to higherdimensional objects in the sense of category theory. In particular, such smooth categorical groups would be valuable as new mathematical objects that would aid in (re)defining and building fundamental physics. One driving notion is that of the string group and its geometric realizations, which are closely tied to the concept of loop groups.

A loop group generalization in this vein has been built in [2], in which a specific relation between Lie 2-algebras and Lie 2-groups was constructed using groups of based paths. This is shown to lead to a geometric realization of the string group. More generally, there is a no-go theorem which states that the 2-group generalization

Ossi Niemimäki

ossi.niemimaki@helsinki.fi

Jouko Mickelsson

jouko@kth.se

1 Department of Mathematics and Statistics, University of Helsinki, P.O. Box 64, FI-00014 Helsinki, Finland 
coming from the direct Lie 2-algebra construction for a simple Lie group $G$ allows for a smooth structure only in a very specific situation [1]. The way around this is to construct an infinite-dimensional Lie 2-group whose Lie 2-algebra is equivalent to the desired Lie 2-algebra.

This approach of using categorical equivalences instead of isomorphisms seems to be crucial in constructing such higher objects. With the string 2-group model in mind, the loop group setting can be extended to quasi-periodic paths [8]. Yet there is a topological obstruction for building a strict 2-group model on this group; the remedy is to flatten the paths around the base point in such a way that there is a categorical equivalence to the original 2-group. This gives a coherent 2-group model for the string group, which — in contrast to the model in [2] — also admits the action of the circle group $S^{1} \cong S O(2)$.

Our aim in this paper is simple: following the strategy from [8], we construct a similar 2-group by considering the group of based maps $\Omega^{3} G$ from the 3-sphere to a Lie group $G$. To begin with, we study the Mickelsson-Faddeev cocycle and the related Lie group extension [6]. This cocycle is crucially dependent on defining Lie algebra valued 1 -forms over the domain-in particular, the extension is not central. ${ }^{1}$

On this 3-loop group extension, we impose the action of the group of smooth maps $B_{b}^{3} G$ from the 3-ball to the Lie group $G$ with the additional condition that the maps are flattened on the boundary $S^{2}$. We show that it is possible to build a crossed module starting with the groups $B_{b}^{3} G$ and $\Omega^{3} G$ in a similar fashion to the loop group described in [8]. The main difference is that the extension of the 3-loop group is not central. This complicates the lifting of the automorphic action $B_{b}^{3} G \rightarrow$ aut $\left(\Omega^{3} G\right)$ to the extension, and furthermore, we cannot define a crossed module directly from these groups. Hence, we need to extend the acting group $B_{b}^{3} G$ by the group $\operatorname{Map}\left(B_{b}^{3} G, S^{1}\right) / S^{1}$. Then, from the automorphic action of $\widehat{B_{b}^{3} G}$ on the 3-loop group extension $\widehat{\Omega^{3} G}$ and the natural projection between these two groups we gain a crossed module and thus a strict 2 group.

One of the strengths of the original loop group construction is that it allows the action of the circle group. For 3-loops, one would like to have the corresponding action of $S O$ (4), but there seems to be no straightforward way to incorporate this into the picture presented here. The extension of the 3-loop group necessitates a fixed point in $B^{3}$-namely, the contracted boundary $S^{2}$-and this choice cannot be equivariant under the symmetry action.

Notation and conventions Unless otherwise stated, $G$ is a simply connected and finitedimensional Lie group. The group identity element is denoted by $e$ throughout, and we write $\mathcal{P}(G)$ for the group of based paths in $G$. The Lie algebra of $G$ is denoted by $\mathfrak{g}$. We identify the 1-sphere $S^{1}$ with the circle group. All the groups of functions taking values in a Lie group are considered as infinite-dimensional Fréchet Lie groups, see [9, Sec. II].

In places, there is an implicit assumption to consider the identity component of a given group in case the connectedness for the whole group is not available.

\footnotetext{
${ }^{1}$ In quantum field theory, the cocycle represents the Hamiltonian quantization anomaly of massless chiral fermions coupled to external gauge potentials.
} 


\section{3-loop group and an automorphic action}

Let $S^{3} G$ be the group of smooth maps from the 3-sphere to a Lie group $G$. Since every $n$-sphere can be given as the quotient $B^{n} / S^{n-1}$ where the boundary of the $n$-ball is contracted to a point, we define by analogy the flattened group

$$
B_{b}^{3} G=\left\{f \in \operatorname{Map}\left(B^{3}, G\right): \partial_{r} f=0 \text { on the boundary } S^{2}\right\},
$$

where the group multiplication is as usual the point-wise multiplication in the domain. We then have the 3-loop group defined as

$$
\Omega^{3} G=\left\{f \in B_{b}^{3} G: f \text { extends to } S^{3} \text { and } f\left(S^{2}\right)=e\right\} .
$$

Note that while the group of rotations $S O(4)$ acts on the sphere group $S^{3} G$, it does not act on $\Omega^{3} G$ since the boundary of $B^{3}$ is the contracted fixed point and the action cannot be extended to $S^{3}$.

It is easy to see that $B_{b}^{3} G$ retains the Lie group structure of $\operatorname{Map}\left(B^{3}, G\right)$, and likewise for $\Omega^{3} G$. In the case of the 1-loop group $\Omega G$ and the free loop group $L G$, there is the split exact sequence

$$
\Omega G \hookrightarrow L G \rightarrow G
$$

For the 3-loop group, this relationship is retained as follows. If we denote by $B_{b, c}^{3} G$ the subgroup of $B_{b}^{3} G$ of maps that are constant on the boundary $S^{2}$, the group $B_{b, c}^{3} G$ is in fact a principal $\Omega^{3} G$-bundle over $G$ :

$$
\Omega^{3} G \hookrightarrow B_{b, c}^{3} G \stackrel{\phi}{\rightarrow} G,
$$

where $\phi^{-1}(e)=\Omega^{3} G$ is the canonical fibre.

Furthermore, we note that $\Omega^{3} G$ is a split normal subgroup of $B_{b}^{3} G$. The quotient map

$$
q: B_{b}^{3} G \rightarrow B_{b}^{3} G / \Omega^{3} G \cong \operatorname{Map}\left(S^{2}, G\right)
$$

has $\Omega^{3} G$ as its kernel.

\subsection{Abelian extension $\widehat{\Omega^{3} G}$ and lifting automorphisms of $\Omega^{3} G$ to its extension}

Given a Fréchet Lie group $\operatorname{Map}(X, G)$ for a manifold $X$, there is an extension by the Abelian group of smooth maps $\operatorname{Map}\left(\operatorname{Map}(X, G), S^{1}\right)$ [11, pp. 66-67]. In particular, we consider an Abelian extension [6]

$$
\operatorname{Map}\left(B_{b}^{3} G, S^{1}\right) \hookrightarrow \widehat{S^{3} G} \rightarrow S^{3} G .
$$


In the gauge theoretical formulation, the fibre is the space of vector potentials $\mathcal{A}$ on $S^{3}$ with values in the Lie algebra $\mathfrak{g}$. The group $\operatorname{Map}\left(B_{b}^{3} G, S^{1}\right)$ is closely related since the potentials $A \in \mathcal{A}$ can be identified with functions $f \in B_{b}^{3} G$ in the sense of Maurer-Cartan forms so that $A:=f^{-1} \mathrm{~d} f .^{2}$

On the Lie algebra level, we have the corresponding Mickelsson-Faddeev cocycle

$$
\theta(A ; x, y)=\frac{i}{24 \pi} \int_{S^{3}} \operatorname{tr} A[\mathrm{~d} x, \mathrm{~d} y] .
$$

No modification is needed to write this in terms of the 3-loop group:

$$
\operatorname{Map}\left(B_{b}^{3} G, S^{1}\right) \hookrightarrow \widehat{\Omega^{3} G} \rightarrow \Omega^{3} G
$$

The corresponding Lie algebra cocycle is essentially of the same form as above.

The right adjoint action $(\cdot)^{f}$ with $f \in B_{b}^{3} G$ adds only a 2-coboundary term to the Lie algebra cocycle:

$$
\theta^{f}=\theta+\{\text { coboundary }\}
$$

One can verify this by direct if a bit tedious computation, see Appendix A. Hence, the cocycle is cohomologically invariant under such automorphisms.

More generally, we have the following proposition.

Proposition 1 Let $K$ be a simply connected Lie group such that $K=H / N$, where $N \subset H$ is a normal subgroup of a simply connected Lie group $H$ for which the first three integral cohomology groups are trivial. Denote by $\pi: H \rightarrow K$ the natural projection so that the fibration has $N$ as the canonical fibre. Then, given a cohomology class $[\omega] \in \mathrm{H}^{3}(K, \mathbb{Z})$, there is a 2 -form $\theta$ on $H$ given by the pullback $\pi^{*} \omega=\mathrm{d} \theta$ such that the following holds:

1. $\theta$ is closed in vertical directions and defines a 2-cocycle on the Lie algebra $\mathfrak{n}$ of $N$ with values in $\operatorname{Map}(H, \mathbb{R})$.

2. $[\theta]$ in $\mathrm{H}^{2}(\mathfrak{n}, \operatorname{Map}(H, \mathbb{R}))$ is uniquely determined by $\omega$.

3. $[\theta]$ in $\mathrm{H}^{2}(\mathfrak{n}, \operatorname{Map}(H, \mathbb{R}))$ is invariant with respect to right action $r_{f}^{*}$, where $f \in H$.

Proof The right action $r_{f}^{*}$ is composed from the right action on the argument in $\operatorname{Map}(H, \mathbb{R})$ and the (right) conjugation on $\mathfrak{n}$. Recall that the Lie algebra of $N$ generates vector fields on $H$ through the right action of $N$. Evaluation of a 2-form $\theta$ (which is closed in vertical directions) along these vector fields gives the 2-cocycle in the Lie algebra $\mathfrak{n}$ with values in smooth functions since the output of any smooth form evaluated along smooth vector fields is a smooth function.

Consider $\theta^{\prime}=\theta+\psi$, where $\psi$ is a closed 2-form. Since $\mathrm{H}^{2}(H, \mathbb{Z})$ is trivial, the 2 -form $\psi$ is also exact. Hence, the 2-cocycle $\theta^{\prime}-\theta$ in $N$ is a coboundary, and $\theta$ defines a 2 -cocycle on the Lie algebra of $N$ with values in $\operatorname{Map}(H, \mathbb{R})$.

${ }^{2}$ See the motivation for the original idea in [6] or [7]. 
Let $f \in H$ and consider a path $f_{t}$ in $H$, where $t \in[0,1]$ with $f(0)=e$ and $f(1)=f$. Then, the cohomology class $[\omega]$ is invariant by the right action in $K$ by $\pi(f)$ :

$$
r_{\pi(f)}^{*} \omega \sim \omega
$$

Moreover,

$$
\mathrm{d}\left(r_{f}^{*} \theta\right)=r_{f}^{*} \mathrm{~d} \theta=r_{\pi(f)}^{*} \omega \sim \omega
$$

Now, given $\omega^{\prime}=\omega+\mathrm{d} \phi$ for some 2 -form $\phi$, we have the pullback

$$
\pi^{*}\left(\omega^{\prime}\right)=\pi^{*} \omega+\mathrm{d}\left(\pi^{*} \phi\right)=\mathrm{d} \theta+\mathrm{d}\left(\pi^{*} \phi\right),
$$

and hence, since the second cohomology group for $H$ is trivial, we can write for some 1 -form $\alpha$

$$
\theta^{\prime}=\theta+\pi^{*} \phi+\mathrm{d} \alpha
$$

where the term $\pi^{*} \phi$ is zero in vertical directions. Thus the Lie algebra cohomology class $[\theta]$ is uniquely determined by $\omega$ and it is invariant with respect to $r_{f}^{*}$ for any $f \in H$.

Remark 1 The proposition can be applied to 3-loops with $K=S^{2} G, N=S_{e}^{3} G$ and $H=\mathcal{P}\left(S^{2} G\right)$. From this, we can squeeze out a general formula for computing the coboundary (see Appendix A).

Remark 2 Since $[\omega] \in \mathrm{H}^{3}(H / N, \mathbb{Z})$, one can think of $\omega$ as a representative of the Dixmier-Douady class on $K$. In the gauge theoretic framework, the group $K$ models the moduli space of based gauge transformations which parameterizes fermionic Fock spaces [12]. There is a natural principal bundle

$$
\mathcal{G}_{e} \hookrightarrow \mathcal{A} \rightarrow \mathcal{A} / \mathcal{G}_{e},
$$

where $\mathcal{A}$ is the space of gauge potentials, and $\mathcal{G}_{e}$ is the space of based gauge transformations related to the symmetry group $G$. The Dixmier-Douady class in $\mathrm{H}^{3}\left(\mathcal{A} / \mathcal{G}_{e}\right)$ represents the obstruction to lifting the $\mathcal{G}_{e}$-bundle to its extension by $\operatorname{Map}\left(\mathcal{A}, S^{1}\right)$. This is also the topological origin of the Lie algebra cocycle (1) [4].

We now the lift the action to the Lie group level while retaining the cohomological invariance of the corresponding 2-cocycle. To this end, we need further results concerning cohomology; more details on the following can be found in [7,10].

Let $H$ be a Lie group. Recall that an Abelian Lie group $A$ is a smooth $H$-module, if it is a $H$-module and the action map $H \times A \rightarrow A$ is smooth. Assuming that $N$ is a normal subgroup of $H$, the group of $N$-invariant elements of $A$ is denoted by 


$$
A^{N}=\{a \in A:(\forall n \in N) n \cdot a=a\}
$$

which is a $H$-submodule of $A$.

Definition 1 (Refined cohomology [10, Appendix D]) Let us denote smooth group cohomology by $\mathrm{H}_{s}^{n}(N, A)$ and continuous Lie algebra cohomology by $\mathrm{H}_{c}^{n}(\mathfrak{n}, \mathfrak{a})$. The gist is that the maps $N^{n} \rightarrow A$ (resp. $\mathfrak{n}^{n} \rightarrow \mathfrak{a}$ ) are smooth (resp. continuous). The smoothness and continuity are defined locally in a neighbourhood of the identity.

A cocycle $f \in Z_{s}^{n}(N, A)$ is smoothly cohomologically invariant with respect to $H$ if there is a map

$$
\phi: H \rightarrow \mathrm{C}_{s}^{p-1}(N, A) \text { such that } \mathrm{d}(\phi(h))=h . f-f \quad \forall h \in H,
$$

and the map

$$
H \times N^{p-1} \rightarrow A:\left(h, n_{1}, \ldots, n_{p-1}\right) \mapsto(h . f-f)\left(n_{1}, \ldots, n_{p-1}\right)
$$

is smooth in an identity neighbourhood. This gives us smoothly invariant cohomology classes of $N$ with values in $A$.

Theorem 1 (Cohomology homomorphism [10, Thm. VII.2]) Let $N$ be a connected Lie group and $A \cong \mathfrak{a} / \Gamma_{A}$ a smooth $N$-module, where $\Gamma_{A} \subset \mathfrak{a}$ is a discrete subgroup of the sequentially complete locally convex space $\mathfrak{a}$. Then, there is an exact sequence

$$
\operatorname{hom}\left(\pi_{1}(N), A^{N}\right) \rightarrow \mathrm{H}_{s}^{2}(N, A) \rightarrow \mathrm{H}_{c}^{2}(\mathfrak{n}, \mathfrak{a}) .
$$

Consider now the right adjoint action of $B_{b}^{3} G$. In order to lift this action, we need to impose $\pi_{1}\left(\Omega^{3} G\right)=0$. In general, it is known that

$$
\pi_{k}\left(S_{e}^{n} G\right) \cong \pi_{k+n}(G)
$$

For instance, if we pick $G=S U(p)$ for $p>2$, each of the connected components of the group $\Omega^{3} G$ is simply connected; for $p=2$, we have topological sectors corresponding to $\mathbb{Z}_{2}$-we discuss this specific case separately at the end of the section. Furthermore, we may restrict the action of $B_{b}^{3} G$ to the identity component of $\Omega^{3} G$ in case non-connectedness becomes an issue.

Assuming $\pi_{4}(G)$ is trivial, $H=B_{b}^{3} G, N=\Omega^{3} G$ and $A=\operatorname{Map}\left(B_{b}^{3} G, S^{1}\right)$ as above, the sequence of Theorem 1 gives a monomorphism $\mathrm{H}_{s}^{2}(N, A) \rightarrow \mathrm{H}_{c}^{2}(\mathfrak{n}, \mathfrak{a})$; the Lie algebra $\mathfrak{a}$ of $A$ is of the form $\operatorname{Map}\left(B_{b}^{3} G, i \mathbb{R}\right)$, with the choice of $\Gamma_{A}=\mathbb{Z}$. In the light of Eq. 2, the Lie algebra cocycle is smoothly cohomologically invariant with respect to right adjoint action by $f \in B_{b}^{3} G$, and by above monomorphism the same holds on the group level.

It is then natural to ask whether we can lift the action of $B_{b}^{3} G$ on $\Omega^{3} G$ to its extension $\widehat{\Omega^{3} G}$. Given any element $f \in B_{b}^{3} G$, we know that there is a smooth action by conjugation both on $\Omega^{3} G$ and the fibre $\operatorname{Map}\left(B_{b}^{3} G, S^{1}\right)$. However, a map from 
$B_{b}^{3} G$ to aut $\left(\widehat{\Omega^{3} G}\right)$ obtained in this fashion is not a group homomorphism; this can be seen by a direct computation already on the Lie algebra level. Generally, we have the following proposition.

Proposition 2 (Lifting homomorphism [7, Prop. 3.8]) Let $H$ be a Lie group, $N$ a connected normal Lie subgroup of $H, \theta \in \mathrm{Z}_{S}^{2}(N, A)$ a smooth 2-cocycle and $\widehat{N}$ the corresponding Lie group extension by an Abelian group A. Then, the smooth group homomorphism $\psi: H \rightarrow \operatorname{aut}(A) \times \operatorname{aut}(N)$ lifts to a smooth homomorphism $\widehat{\psi}: H \rightarrow \operatorname{aut}(\widehat{N}, A)$ if and only if

1. $\theta$ is smoothly cohomologically invariant with respect to $H$, and

2. the corresponding cohomology class $\left[\mathrm{d}_{\psi} \phi\right] \in \mathrm{H}_{s}^{2}\left(H, \mathrm{Z}_{s}^{1}(N, A)\right)$ is trivial, where the 1-cocycle $\phi$ is defined via $\mathrm{d}_{N}(\phi(h))=h . \theta-\theta$ for any $h \in H$.

The group aut $(\widehat{N}, A)$ in Proposition 2 is formed from automorphisms of the extension $\widehat{N}$ that preserve the split Lie subgroup $A$. The $\operatorname{map~}_{\psi}$ is the coboundary operator in the cochain complex of maps $f: H^{p} \rightarrow \mathrm{C}_{s}^{1}(N, A)$, defined in relation to the action of the group $H$ on $\mathrm{C}_{s}^{1}(N, A)$ by $h . f=\psi(h) . f$ (in [7], the corresponding notation is $\mathrm{d}_{S_{\psi}}$, where $S_{\psi}$ denotes the action).

The first condition is fulfilled in the case of the 3-loop group, but the cohomology class given by $\mathrm{d}_{\psi} \phi$ is not trivial, and thus, the action of $B_{b}^{3} G$ does not lift to the extension. However, keeping in mind that our goal is a crossed module, we can reroute our approach through central extensions as will be explained in the next section. Let us conclude by gathering the results of this section in the following proposition:

Proposition 3 Let $\pi_{1}\left(\Omega^{3} G\right)$ be trivial. Then, the 2-cocycle $\theta$ of the Abelian Lie group extension

$$
\operatorname{Map}\left(B_{b}^{3} G, S^{1}\right) \hookrightarrow \widehat{\Omega^{3} G} \rightarrow \Omega^{3} G
$$

is smoothly cohomologically invariant with respect to the right adjoint action of $B_{b}^{3} G$. However, the action of $B_{b}^{3} G$ on $\Omega^{3} G$ does not lift to an automorphic action on $\widehat{\Omega^{3} G}$.

\subsection{The case of $S U(2)$}

For $G=S U(2) \cong S^{3}$, we have $\pi_{4}(G)=\mathbb{Z}_{2}$. Moreover, in this case the above Lie algebra cocycle is identically zero [6]. There is, however, a non-trivial extension

$$
\mathbb{Z}_{2} \hookrightarrow \mathcal{G} \rightarrow \Omega^{3} G
$$

where $\mathcal{G}$ is the group of homotopy classes of paths $f$ in $\Omega^{3} G$ such that $f(0)=e$ and $f(1)=g$, when we consider only the identity component. This is a covering group for the component of the identity in $\Omega^{3} G$, and there is a natural projection to path points.

The right conjugation by $f \in B_{b}^{3} G$ acting on any given path point does not change the homotopy. Thus, the right adjoint action of the group $B_{b}^{3} G$ on $\Omega^{3} G$ lifts to an automorphic action on the extension $\mathcal{G}$. 


\section{2-group from a crossed module}

The aim of this paper to is construct an action groupoid that would fulfil the axioms of a crossed module, and thus define a strict 2-group [3]. Let us revisit the definitions.

Definition 2 (Crossed module) Let $G$ and $H$ be groups, and consider morphisms

$$
\delta: H \rightarrow G \text { and } \alpha: G \rightarrow \operatorname{aut}(H)
$$

We say that $[\delta: H \rightarrow G]$ is a crossed module if the following two diagrams commute.

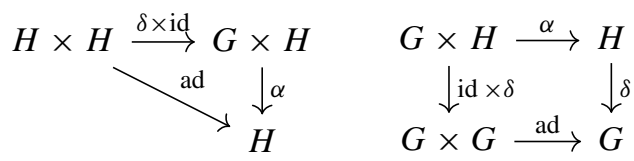

Equivalently, if we denote by $h^{g}$ the element-wise action of $G$ on $H$, the diagrams correspond to the equations

$$
h^{\delta\left(h^{\prime}\right)}=h^{\prime-1} h h^{\prime}
$$

and

$$
\delta\left(h^{g}\right)=g^{-1} \delta(h) g
$$

for all $h, h^{\prime} \in H$ and $g \in G$.

Definition 3 (Smooth crossed module) If the groups $G$ and $H$ in a crossed module $[\delta: H \rightarrow G]$ are Lie groups and the action defined by the morphism $\alpha$ is smooth, the crossed module is called a Lie crossed module, or a smooth crossed module.

As stated in Proposition 3, we do not have a lift of the action of $B_{b}^{3} G$ to the extension $\widehat{\Omega^{3} G}$. Even if we had, this would not be sufficient for constructing a crossed module, since the extension $\widehat{\Omega^{3} G}$ would not allow for a natural morphism $\delta$ to the acting group $B_{b}^{3} G$. Let us then extend the group $B_{b}^{3} G$ as follows. There is a topologically trivial Lie group extension

$$
\operatorname{Map}\left(B_{b}^{3} G, S^{1}\right) / S^{1} \hookrightarrow \widehat{B_{b}^{3} G} \rightarrow B_{b}^{3} G
$$

which on the Lie algebra level links back to the Mickelsson-Faddeev cocycle (modulo the Lie algebra of $S^{1}$ ). The reason for modifying the centre with $S^{1}$ comes from the need to fulfil the cocycle condition when considering the domain $B^{3}$ instead of $S^{3}$ : there is a constant boundary term

$$
\delta \theta=\frac{i}{24 \pi} \int_{\partial B^{3}} \operatorname{tr} x[\mathrm{~d} y, \mathrm{~d} z]-y[\mathrm{~d} z, \mathrm{~d} x]+z[\mathrm{~d} x, \mathrm{~d} y],
$$


which then vanishes by construction since the Lie algebra of $S^{1}$ is $i \mathbb{R}$.

Note that now the right adjoint action of $\widehat{B_{b}^{3} G}$ defines automorphisms on the Lie algebra of the extension $\widehat{\Omega^{3} G}$ so that we have a homomorphism

$$
\widehat{B_{b}^{3} G} \rightarrow \operatorname{aut}\left(\widehat{\Omega^{3} \mathfrak{g}}\right)
$$

which leads to the following proposition.

Proposition 4 If the (identity component of the) group $\widehat{\Omega^{3} G}$ is simply connected, then the homomorphism

$$
\alpha^{\prime}: \widehat{B_{b}^{3} G} \rightarrow \operatorname{aut}\left(\widehat{\left.\Omega^{3} \mathfrak{g}\right)}\right.
$$

lifts to a homomorphism

$$
\alpha: \widehat{B_{b}^{3} G} \rightarrow \operatorname{aut}\left(\widehat{\Omega^{3} G}\right)
$$

such that the action on $\widehat{\Omega^{3} G}$ is smooth.

Proof The statement is a direct consequence of Corollary 9.5.11 in [5, p. 341], which gives a Lie group isomorphism between the automorphism groups of a connected and simply connected Lie group and its Lie algebra. Hence, the following diagram commutes, and the action given by the map $\alpha$ is smooth.

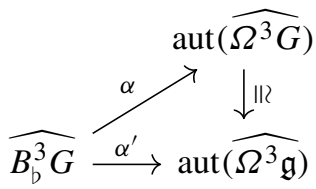

Remark 3 For the canonical central extension of the 1-loop group $\Omega G$, it holds that $\pi_{1} \widehat{(\Omega G)}=0$. A similar argument based on the long homotopy exact sequence can be used in the case of $\Omega^{3} G$.

On the other hand, whether $\widehat{\Omega^{3} G}$ is simply connected or not, we can consider the group

$$
\mathcal{S}=\operatorname{Map}\left(B_{b}^{3} G, S^{1}\right) / S^{1} \rtimes \Omega^{3} G
$$

which is a normal subgroup of $\widehat{B_{b}^{3} G}$ since $\Omega^{3} G$ is normal in $B_{b}^{3} G$ and the fibre $\operatorname{Map}\left(B_{b}^{3} G, S^{1}\right) / S^{1}$ is mapped onto itself in conjugation. Hence, there is a smooth right adjoint action of $\widehat{B_{b}^{3} G}$ on $\mathcal{S}$ by conjugation. Furthermore, while the previously introduced 3-loop group extension is not central, there is a central extension

$$
S^{1} \hookrightarrow \widehat{\mathcal{S}} \rightarrow \mathcal{S}
$$


where the group $\widehat{B_{b}^{3} G}$ acts trivially on the central $S^{1}$. Note that this is none other than the original extension $\widehat{\Omega^{3} G}$ of the 3-loop group.

Now, we can consider the right adjoint action of $\widehat{B_{b}^{3} G}$ on the group $\widehat{\Omega^{3} G}$. The cocycle corresponding to $\widehat{\Omega^{3} G}$ is the familiar 2-cocycle $\theta$, and so by Proposition 3 and the construction above we know that it is smoothly cohomologically invariant with respect to the action of $\widehat{B_{b}^{3} G}$. Hence, the first condition of Proposition 2 for lifting the homomorphism

$$
\psi: \widehat{B_{b}^{3} G} \rightarrow \operatorname{aut}\left(S^{1}\right) \times \operatorname{aut}\left(\operatorname{Map}\left(B_{b}^{3} G, S^{1}\right) / S^{1} \rtimes \Omega^{3} G\right)
$$

to a homomorphism

$$
\widehat{\psi}: \widehat{B_{b}^{3} G} \rightarrow \operatorname{aut}\left(\widehat{\Omega^{3} G}, \operatorname{Map}\left(B_{b}^{3} G, S^{1}\right)\right)
$$

is fulfilled.

For the second condition, we observe the following. Let groups $H, N$ and $A$ be as in Proposition 2 with the cocycle $\theta \in \mathrm{Z}_{S}^{2}(N, A)$ corresponding to the Abelian extension $\widehat{N}$. Assuming that $N$ is split normal subgroup of $H$ and that $A^{N}$ is a split Lie subgroup of $A$, there is a commuting diagram [7, Lemma 4.3 and the preceding definitions]

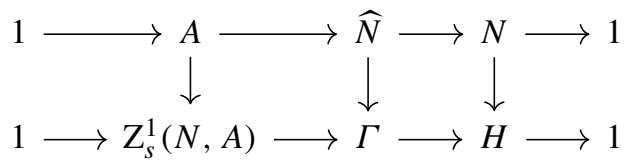

where $\Gamma$ is the extension of $H$ by $\mathrm{Z}_{S}^{1}(N, A)$. Furthermore, there is a smooth automorphic action $\Gamma \rightarrow$ aut $(\widehat{N})$; the pair $(\widehat{N}, \Gamma)$ in fact has the structure of a smooth crossed module provided that the cohomology group $\mathrm{H}_{s}^{1}(N, A)$ is trivial [7, Prop. 4.5 and Thm. 4.6]. In particular, this condition ensures that $\Gamma$ is a Lie group extension and that $Z_{S}^{1}(N, A) \cong A / A^{N}$.

Lemma 1 Let $G=S U(p)$ with $p \geq 3$. Then, the first cohomology group $\mathrm{H}_{s}^{1}\left(\Omega^{3} G, \operatorname{Map}\left(B_{b}^{3} G, S^{1}\right)\right)$ is trivial.

Proof Denote $N=\Omega^{3} G, A=\operatorname{Map}\left(B_{b}^{3} G, S^{1}\right)$ and $H=\operatorname{Map}\left(S^{2}, G\right)$; note that we can identify $H$ with $B_{b}^{3} G / N$. By the Bott periodicity, the cohomology of $H$ in low degrees is generated by elements $\alpha_{2 k+1}$ in odd degrees $(k=1,2, \ldots)$. In particular, $\mathrm{H}_{s}^{2}(H, \mathbb{Z})$ vanishes for $p \geq 3$ and there are no non-trivial circle bundles over $H$.

On the other hand, an element $c_{1} \in \mathrm{H}_{s}^{1}(N, A)$ describes a circle bundle $Q$ over $H=B_{b}^{3} G / N$ : elements in $Q$ are equivalence classes of pairs $(g, \lambda) \in B_{b}^{3} G \times S^{1}$ with the equivalence relation $(g, \lambda) \sim\left(g u, c_{1}(g ; u) \lambda\right)$. The bundle can be trivialized if and only if $c_{1}(g ; u)=f(g u) f(g)^{-1}$ for some $f: B_{b}^{3} G \rightarrow S^{1}$. Thus, the group $\mathrm{H}_{S}^{1}(N, A)$ is trivial. 
Assuming that we have the conditions satisfying the preceding Lemma, there is then a smooth homomorphism

$$
\Gamma \rightarrow \operatorname{aut}\left(\widehat{\Omega^{3} G}\right)
$$

where the Lie group $\Gamma$ is defined by the extension

$$
\operatorname{Map}\left(B_{b}^{3} G, S^{1}\right) / \operatorname{Map}\left(S^{2} G, S^{1}\right) \hookrightarrow \Gamma \rightarrow B_{b}^{3} G .
$$

Here, we have identified $A^{N}:=\operatorname{Map}\left(B_{b}^{3} G, S^{1}\right)^{\Omega^{3} G}$ with $\operatorname{Map}\left(S^{2} G, S^{1}\right)$.

We further note that there is a natural smooth homomorphism

$$
p: \operatorname{Map}\left(B_{b}^{3} G, S^{1}\right) / S^{1} \rightarrow \operatorname{Map}\left(B_{b}^{3} G, S^{1}\right) / \operatorname{Map}\left(S^{2} G, S^{1}\right),
$$

and that the fibre quotient $\operatorname{Map}\left(S^{2} G, S^{1}\right)$ acts trivially on $\widehat{\Omega^{3} G}$. Hence, the map $\Gamma \rightarrow \operatorname{aut}\left(\widehat{\Omega^{3} G}\right)$ lifts to a smooth homomorphism

$$
\alpha: \widehat{B_{b}^{3} G} \rightarrow \operatorname{aut}\left(\widehat{\Omega^{3} G}\right)
$$

which gives us a unique action

$$
\widehat{\Omega^{3} G} \times \widehat{B_{b}^{3} G} \rightarrow \widehat{\Omega^{3} G}
$$

This defines an action groupoid $\widehat{\Omega^{3} G} / / \widehat{B_{b}^{3} G}$.

Let us recap what we have done. We started with the Abelian extension

$$
\operatorname{Map}\left(B_{b}^{3} G, S^{1}\right) \hookrightarrow \widehat{\Omega^{3} G} \rightarrow \Omega^{3} G
$$

with the intention of replicating the 1-loop group crossed module of [8] for 3-loops in $G$ by using the action of $B_{b}^{3} G$ on $\Omega^{3} G$. However, this action does not lift to the extension $\widehat{\Omega^{3} G}$, and there is no natural morphism $\widehat{\Omega^{3} G} \rightarrow B_{b}^{3} G$ which would satisfy the requirements of a crossed module. It is then clear that we must take two additional steps: first by extending the acting group $B_{b}^{3} G$ as

$$
\operatorname{Map}\left(B_{b}^{3} G, S^{1}\right) / S^{1} \hookrightarrow \widehat{B_{b}^{3} G} \rightarrow B_{b}^{3} G
$$

and second by imposing this action on the central extension of Eq. 3

$$
S^{1} \hookrightarrow \widehat{\mathcal{S}} \rightarrow \mathcal{S}
$$

where $\mathcal{S}$ equals to $\operatorname{Map}\left(B_{b}^{3} G, S^{1}\right) / S^{1} \rtimes \Omega^{3} G$. 
Now for the smooth crossed module of Definition 3, we need morphisms $\delta$ : $\widehat{\Omega^{3} G} \rightarrow \widehat{B_{b}^{3} G}$ and $\alpha: \widehat{B_{b}^{3} G} \rightarrow$ aut $\left(\widehat{\Omega^{3} G}\right)$ (where $\alpha$ is smooth). The action groupoid $\widehat{\Omega^{3} G} / \widehat{B_{b}^{3} G}$ fulfils these requirements by construction. Let $\alpha: \widehat{B_{b}^{3} G} \rightarrow \operatorname{aut}\left(\widehat{\Omega^{3} G}\right)$ be as above. The morphism $\delta: \widehat{\Omega^{3} G} \rightarrow \widehat{B_{b}^{3} G}$ is gathered as a composite of the epimorphism in the central extension (3) and the natural inclusion:

$$
\widehat{\Omega^{3} G} \rightarrow \operatorname{Map}\left(B_{b}^{3} G, S^{1}\right) / S^{1} \rtimes \Omega^{3} G \hookrightarrow \widehat{B_{b}^{3} G}
$$

This strict 2-group is then a 3-loop analogue to that in [8, Definition 3.5.]. In contrast, we have no comparable equivalence of Lie groupoids ([8, Theorem 3.6.]), since there is no analogue to the non-smooth quasi-periodic action. Likewise, there seems to be no way to include the $S O(4)$-equivariance in this picture.

Acknowledgements Open access funding provided by University of Helsinki including Helsinki University Central Hospital. We wish to thank David M. Roberts for his comments and helpful criticism. This research was partially supported by the Emil Aaltonen Foundation (Grant No. 170185 N) and the Niilo Helander Foundation.

Open Access This article is distributed under the terms of the Creative Commons Attribution 4.0 International License (http://creativecommons.org/licenses/by/4.0/), which permits unrestricted use, distribution, and reproduction in any medium, provided you give appropriate credit to the original author(s) and the source, provide a link to the Creative Commons license, and indicate if changes were made.

\section{A Cohomological conjugation invariance of the Mickelsson-Faddeev cocycle}

\section{A.1 Explicit coboundary terms for the conjugated 2-cocycle}

Consider the following 2-cocycle for the Lie algebra $\Omega^{3} \mathfrak{g}$ :

$$
\theta(A ; x, y)=\int_{S^{3}} \operatorname{tr} A[\mathrm{~d} x, \mathrm{~d} y]
$$

We wish to show that the cocycle is cohomologically invariant under the right adjoint action of the group $B_{b}^{3} G$.

On the Lie algebra, the action is the right conjugation by $f \in B_{b}^{3} G$ :

$$
A \mapsto f^{-1} A f+f^{-1} \mathrm{~d} f, \quad x \mapsto f^{-1} x f,
$$

so that

$$
\mathrm{d}\left(x^{f}\right)=-f^{-1} \omega x f+f^{-1} \mathrm{~d} x f+f^{-1} x \mathrm{~d} f,
$$


where $\omega:=\mathrm{d} f f^{-1}$ is the right-invariant Maurer-Cartan form. We then get the conjugated cocycle $\theta^{f}$ :

$$
\begin{aligned}
& \int_{S^{3}} \operatorname{tr} A[\mathrm{~d} x, \mathrm{~d} y]-A \omega[x, y] \omega+A\left(x \omega \mathrm{d} y-y \omega \mathrm{d} x-x \omega^{2} y+y \omega^{2} x-\mathrm{d} x \omega y+\mathrm{d} y \omega x\right) \\
& \quad+(A \omega+\omega A)(x \omega y-y \omega x)-A \omega(x \mathrm{~d} y-y \mathrm{~d} x)+\omega A(\mathrm{~d} x y-\mathrm{d} y x) \\
& \quad+2 \omega^{2}(x \omega y-y \omega x)-\omega\left(\mathrm{d} x \omega y-\mathrm{d} y \omega x+x \omega^{2} y-y \omega^{2} x-x \omega \mathrm{d} y+y \omega \mathrm{d} x\right) \\
& +\omega^{2}(\mathrm{~d} x y-\mathrm{d} y x-x \mathrm{~d} y-y \mathrm{~d} x)+\omega[\mathrm{d} x, \mathrm{~d} y]-\omega^{3}[x, y] .
\end{aligned}
$$

Using $\omega^{2}=\mathrm{d} \omega$ and Stokes' theorem, we can reduce the terms independent of $A$ to:

$$
\omega^{2}(x \omega y-y \omega x)-\omega[\mathrm{d} x, \mathrm{~d} y]-\omega^{3}[x, y]
$$

Let $\lambda(A ; z)$ be a 1 -cochain. Then,

$$
\delta(\lambda)(A ; x, y)=\mathcal{L}_{x} \lambda(A ; y)-\mathcal{L}_{y} \lambda(A ; x)-\lambda(A ;[x, y]),
$$

where the derivation $\mathcal{L}_{x}$ acts on $A$ as

$$
\mathcal{L}_{x} A=[A, x]+\mathrm{d} x
$$

Let $\lambda_{i}(A ; z)$ be 1 -cochains as follows:

$$
\begin{aligned}
& \lambda_{1}(A ; z)=\int_{S^{3}} \operatorname{tr} A \omega[\omega, z], \\
& \lambda_{2}(A ; z)=\int_{S^{3}} \operatorname{tr}[\omega, A] \mathrm{d} z, \\
& \lambda_{3}(A ; z)=\int_{S^{3}} \operatorname{tr} \omega^{3} z .
\end{aligned}
$$

The coboundaries are

$$
\begin{aligned}
\delta \lambda_{1}(A ; x, y)= & \int_{S^{3}} \operatorname{tr} A\left(x \omega^{2} y-y \omega^{2} x\right)-\omega^{2}(x \mathrm{~d} y-y \mathrm{~d} x) \\
& +A \omega[x, y] \omega-(A \omega+\omega A)(x \omega y-y \omega x)+\omega(x \omega \mathrm{d} y-y \omega \mathrm{d} x), \\
\delta \lambda_{3}(A ; x, y)= & \int_{S^{3}} \operatorname{tr} A(\mathrm{~d} x \omega y-\mathrm{d} y \omega x)-\omega A(\mathrm{~d} x y-\mathrm{d} y x) \\
& -A(x \omega \mathrm{d} y-y \omega \mathrm{d} x)+A \omega(x \mathrm{~d} y-y \mathrm{~d} x)+2 \omega[\mathrm{d} x, \mathrm{~d} y], \\
\delta \lambda_{3}(A ; x, y)= & \int_{S^{3}} \operatorname{tr} \omega^{3}[x, y] .
\end{aligned}
$$


Then, clearly

$$
\theta^{f}=\theta-\sum_{i} \delta \lambda_{i}
$$

as wanted.

\section{A.2 General formula}

Let us expand on Proposition 1 and Remark 1. Assume $f, g \in \mathcal{P}\left(S^{2} G\right)$ and consider 2-cocycle $\omega(g ; x, y)$ for the Lie algebra of $\Omega\left(S^{2} G\right)$. We have the right action

$$
\omega^{f}(g ; x, y)=\omega\left(g f ; \operatorname{ad}_{f}(x), \operatorname{ad}_{f}(y)\right),
$$

where the adjoint action is $\operatorname{ad}_{f}(x)=f^{-1} x f$ in a matrix representation. We wish to show that $\omega^{f}-\omega$ is a coboundary. The argument is essentially the same as in the Poincaré lemma.

Let $f_{t}$ be a path in $\mathcal{P}\left(S^{2} G\right)$ with $f_{0}=e$ and $f_{1}=f$. Let us then rewrite the sought term as follows:

$$
\begin{aligned}
& \omega^{f}-\omega=\int_{0}^{1} \frac{\mathrm{d}}{\mathrm{d} t} \omega^{f} \mathrm{~d} t \\
& =\int_{0}^{1} \omega\left(g \dot{f}_{t} ; \operatorname{ad}_{f_{t}} x, \operatorname{ad}_{f_{t}} y\right) \mathrm{d} t+\int_{0}^{1} \omega\left(g f_{t} ;\left[f_{t}^{-1} x f_{t}, f_{t}^{-1} \dot{f}_{t}\right], f_{t}^{-1} y f_{t}\right) \mathrm{d} t \\
& +\int_{0}^{1} \omega\left(g f_{t} ; f_{t}^{-1} x f_{t},\left[f_{t}^{-1} y f_{t}, f_{t}^{-1} \dot{f}_{t}\right]\right) \mathrm{d} t \\
& =-\int_{0}^{1} \omega\left(g f_{t} ; f_{t}^{-1} \dot{f}_{t},\left[f_{t}^{-1} x f_{t}, f_{t}^{-1} y f_{t}\right]\right) \mathrm{d} t \\
& +\int_{0}^{1} \mathcal{L}_{f_{t}-1} x f_{t} \omega\left(g f_{t} ; f_{t}^{-1} \dot{f}_{t}, f_{t}^{-1} y f_{t}\right) \mathrm{d} t \\
& +\int_{0}^{1} \mathcal{L}_{f_{t}^{-1} y f_{t}} \omega\left(g f_{t} ; f_{t}^{-1} \dot{f}_{t}, f_{t}^{-1} x f_{t}\right) \mathrm{d} t
\end{aligned}
$$

where on the last equality we have used the 2-cocycle property of $\omega$. The result indeed is a coboundary $(\delta \theta)(g ; x, y)$, where

$$
\theta(g ; z)=\int_{0}^{1} \omega\left(g f_{t} ; f_{t}^{-1} \dot{f}_{t}, f_{t}^{-1} z f_{t}\right) \mathrm{d} t .
$$

It is important that either $x$ or $y$ must be periodic (hence $\Omega\left(S^{2} G\right)$ so that both are), for this ensures that the boundary terms vanish.

Let us sketch this more concretely for the one-dimensional loops with the known 2-cocycle 


$$
\omega(x, y)=\frac{1}{2 \pi} \int_{0}^{2 \pi} \operatorname{tr} x y^{\prime} \mathrm{d} \phi
$$

in the Lie algebra $\Omega \mathfrak{g}$. Here, we have abbreviated $\frac{\mathrm{d}}{\mathrm{d} \phi} \alpha=\alpha^{\prime}$ in contrast to the pathrelated derivation $\frac{\mathrm{d}}{\mathrm{d} t} \alpha=\dot{\alpha}$. Now,

$$
\begin{aligned}
\theta(x) & =\int_{0}^{1} \omega\left(f_{t}^{-1} \dot{f}_{t}, f_{t}^{-1} x f_{t}\right) \mathrm{d} t \\
& =\frac{1}{2 \pi} \int_{0}^{1} \int_{0}^{2 \pi} \operatorname{tr} f_{t}^{-1} \dot{f}_{t} \frac{\mathrm{d}}{\mathrm{d} \phi}\left(f_{t}^{-1} x f_{t}\right) \mathrm{d} \phi \mathrm{d} t .
\end{aligned}
$$

We can choose a path $f_{t}=e^{t z(\phi)}$, where $z:[0,2 \pi] \rightarrow \mathfrak{g}$. We then have

$$
f_{t}^{-1} \dot{f}_{t}=z(\phi)
$$

and so for the cochain

$$
\begin{aligned}
\theta(x) & =\frac{1}{2 \pi} \int_{0}^{1} \int_{0}^{2 \pi} \operatorname{tr} z(\phi) \frac{\mathrm{d}}{\mathrm{d} \phi}\left(f_{t}^{-1} x f_{t}\right) \mathrm{d} \phi \mathrm{d} t \\
& =-\frac{1}{2 \pi} \int_{0}^{1} \int_{0}^{2 \pi} \operatorname{tr} z^{\prime}(\phi)\left(f_{t}^{-1} x f_{t}\right) \mathrm{d} \phi \mathrm{d} t \\
& =-\frac{1}{2 \pi} \int_{0}^{1} \int_{0}^{2 \pi} \operatorname{tr} f_{t} z^{\prime}(\phi) f_{t}^{-1} x \mathrm{~d} \phi \mathrm{d} t
\end{aligned}
$$

by partial integration and the cyclicity of the trace. Next, we note that

$$
\frac{\mathrm{d}}{\mathrm{d} t}\left(f_{t} \frac{\mathrm{d}}{\mathrm{d} \phi} f_{t}^{-1}\right)=f_{t} z(\phi) \frac{\mathrm{d}}{\mathrm{d} \phi} f_{t}^{-1}-f_{t} \frac{\mathrm{d}}{\mathrm{d} \phi}\left(z(\phi) f_{t}^{-1}\right)=-f_{t} z^{\prime}(\phi) f_{t}^{-1}
$$

Inserting this back to the cochain, we have

$$
\theta(x)=\frac{1}{2 \pi} \int_{0}^{1} \int_{0}^{2 \pi} \operatorname{tr} \frac{\mathrm{d}}{\mathrm{d} t}\left(f_{t} \frac{\mathrm{d}}{\mathrm{d} \phi} f_{t}^{-1}\right) x \mathrm{~d} \phi \mathrm{d} t
$$

so that finally by integrating over $t$ we see that our cochain indeed is a coboundary [7, Example 3.10]

$$
\theta(x)=-\frac{1}{2 \pi} \int_{0}^{2 \pi} \operatorname{tr} f_{1}^{\prime} f_{1}^{-1} x \mathrm{~d} \phi
$$

\section{References}

1. Baez, J.C., Lauda, A.D.: Higher-dimensional algebra v: 2-groups. Theory Appl. Categ. 12, 423-491 (2004) 
2. Baez, J.C., Stevenson, D., Crans, A.S., Schreiber, U.: From loop groups to 2-groups. Homol. Homotopy Appl. 9(2), 101-135 (2007)

3. Brown, R., Spencer, C.B.: $G$-groupoids, crossed modules and the fundamental groupoid of a topological group. Ned. Akad. Wet. Proc. Ser. A 79(4), 296-302 (1976)

4. Carey, A., Mickelsson, J., Murray, M.: Index theory, gerbes, and Hamiltonian quantization. Commun. Math. Phys. 183(3), 707-722 (1997)

5. Hilgert, J., Neeb, K.H.: Structure and Geometry of Lie Groups. Springer Monographs in Mathematics. Springer, New York (2012)

6. Mickelsson, J.: Kac-Moody groups, topology of the dirac determinant bundle, and fermionization. Commun. Math. Phys. 110(2), 173-183 (1987)

7. Mickelsson, J., Wagner, S.: Third group cohomology and gerbes over Lie groups. J. Geom. Phys. 108, 49-70 (2016)

8. Murray, M., Roberts, D.M., Wockel, C.: Quasi-periodic paths and a string 2-group model from the free loop group. J. Lie Theory 27(4), 1151-1177 (2017)

9. Neeb, K.H.: Borel-Weil theory for loop groups. In: Infinite Dimensional Kähler Manifolds. Springer, pp. 179-229 (2001)

10. Neeb, K.H.: Abelian extensions of infinite-dimensional lie groups. In: Travaux mathématiques. Fasc. XV, Trav. Math., vol. 15, pp. 69-194 (2004)

11. Pressley, A., Segal, G.: Loop Groups. Oxford Mathematical Monographs. The Clarendon Press, New York (1986)

12. Singer, I.M.: The geometry of the orbit space for non-Abelian gauge theories. Phys. Scr. 24(5), 817-820 (1981)

Publisher's Note Springer Nature remains neutral with regard to jurisdictional claims in published maps and institutional affiliations. 